\title{
PERFIL EPIDEMIOLÓGICO DOS ÓBITOS POR DOENÇA DE CHAGAS NO ESTADO DO TOCANTINS ENTRE 2008 E 2018.
}

\section{EPIDEMIOLOGICAL PROFILE OF DEATHS FROM CHAGAS DISEASE IN THE STATE OF TOCANTINS BETWEEN 2008 AND 2018.}

\author{
Euber Joe Jurado Martinez ${ }^{1}$, Bruna Soares de Sousa ${ }^{1}$, Higor Vinícius Rocha \\ Faria ${ }^{1}$, Bruno Garcia Simões Favaretto ${ }^{2}$.
}

\begin{abstract}
RESUMO
Objetivo: Este estudo tem o desígnio de averiguar e traçar o Perfil Epidemiológico dos óbitos causados pela Doença de Chagas no Estado do Tocantins no período de 2008 a 2018. Método: Trata-se de uma pesquisa longitudinal, qualitativa e quantitativa, baseada em dados obtidos a partir do DATASUS e IBGE para cada município do Tocantins, entre 2008 e 2018. Os dados coletados foram: número absoluto de óbitos relacionados à Doença de Chagas; número de médicos; número de agentes comunitários de saúde (ACS); e quantidade de Unidades de Vigilância em Saúde (UVS). Os óbitos foram obtidos discriminadamente, conforme município de residência, para: cada ano; sexo; etnia; faixa etária; níveis de escolaridade e local de registro. E a informação acerca da população beneficiária de Planos de Saúde no Tocantins foi acessada via ANS. Resultados: Os óbitos analisados no período de 2008 a 2018 foi de 578. Observando a variável sexo, nota-se um predomínio do sexo masculino com $60 \%$. No que diz respeito a cor de pele, a cor parda lidera com $57,96 \%$. A faixa etária mais acometida foi entre 70 e 79 anos com 169 óbitos. A partir de 4 a 7 anos de tempo de estudo a incidência diminui progressivamente na medida que a escolaridade aumenta. Levando em consideração o local de ocorrência, a maioria aconteceu no hospital $(67,82 \%)$. Por fim, a Macrorregião de Saúde mais afetada foi a Sudeste. Conclusões: O perfil, portanto, dos óbitos por Chagas no Tocantins entre 2008 e 2018 é composto por homens da raça parda, entre 70 e 79 anos. Mais de $67 \%$ dos óbitos ocorreram nos hospitais. Além disso, ao comparar os óbitos no Tocantins com os demais estados do país, nota-se a necessidade de elaboração de programas de saúde, devendo-se aplicar as informações demonstradas por este estudo para subsidiar essas condutas de saúde pública.
\end{abstract}

Palavras-chave: Doença de Chagas; Perfil de Saúde; Mortalidade.

\begin{abstract}
Autor correspondente: Euber Joe Jurado Martinez. 606 Norte, Alameda A, QI 03, Lote 03, Plano Diretor Norte, Palmas, Tocantins. CEP: 77006-778. E-mail: euberijm04@hotmail.com
\end{abstract}

Editor: Rosa A. C. g. Medicina, Universidade Federal do Tocantins, Brasil.

Publicado: 25 de julho de 2021.

Direitos Autorais: (c) 2021 Martinez et al. Este é um artigo de acesso aberto que permite o uso, a distribuição e a reprodução sem restrições em qualquer meio, desde que o autor original e a fonte sejam creditados.

Conflito de interesses: os autores declararam que não existem conflitos de interesses.

\begin{abstract}
Objective: This study aims to investigate and trace the Epidemiological Profile of deaths caused by Chagas Disease in the State of Tocantins from 2008 to 2018. Methods: This is a longitudinal, qualitative and quantitative research, based on data obtained from DATASUS and IBGE for each municipality in Tocantins, between 2008 and 2018. The data collected were: absolute number of deaths related to Chagas disease; number of doctors; number of community health agents (ACS); and number of Health Surveillance Units (UVS). The deaths were obtained separately, according to the municipality of residence, for: each year; sex; ethnicity; age group; schooling levels and place of registration. Information about the beneficiary population of Health Plans in Tocantins was accessed via ANS. Results: The deaths analyzed in the period from 2008 to 2018 were 578 . Observing the gender variable, there is a predominance of males with $60 \%$. With regard to skin color, the brown color leads with $57.96 \%$. The most affected age group was between 70 and 79 years old with 169 deaths. From 4 to 7 years of study time, the incidence decreases progressively as schooling increases. Taking into account the place of occurrence, most occurred in the hospital (67.82\%). Finally, the Health Macroregion most affected was the Southeast. Conclusion: The profile, therefore, of deaths by Chagas in Tocantins between 2008 and 2018 is composed of men of the brown race, between 70 and 79 years old. More than $67 \%$ of deaths occurred in hospitals. In addition, when comparing deaths in Tocantins with other states in the country, there is a need for the elaboration of health programs, and the information shown in this study should be applied to support these public health practices.

Keywords: Chagas Disease; Epidemiology; Mortality.
\end{abstract}


pacientes, diminuindo as mortes evitáveis através de diagnóstico e tratamento precoce.

A Doença de Chagas é uma doença infecciosa considerada uma enfermidade negligenciada pela Organização Mundial da Saúde ${ }^{1,2}$, porém possuí elevada morbimortalidade e grandes impactos psicossocial e econômico ${ }^{3}$. Essa patologia é conhecida como Tripanossomíase americana, pois é comum na América do Sul, em especial, no Brasil ${ }^{4}$. Causada pelo protozoário Trypanosoma cruzi, infecta além dos seres humanos, diversos animais como cães, gatos e roedores. A transmissão entre animais e humanos acontece por vetores triatomídeos (barbeiro, chupão, procotó ou bicudo) ${ }^{4,5}$.

A doença pode cursar em fase aguda (sintomática, de sintomas leves de síndrome febril e edema) ${ }^{5}$ ou crônica (de manifestação indeterminada, acometendo os sistemas cardíaco e/ou gastrointestinal) ${ }^{5,6}$. Na Doença de Chagas, especialmente devido às complicações da fase crônica, o dano causado às células miocárdicas e às células nervosas das vias de condução podem resultar em óbito ou outras morbidades potencialmente fatais ${ }^{4}$. Apesar dos diferentes cenários e epidemiologias regionais, por representar um importante problema de saúde pública no $\mathrm{Brasil}^{3}$, a Doença de Chagas possui notificação compulsória no país ${ }^{7}$. Além disso, o governo disponibiliza material prático de orientação para diagnóstico e manejo de casos de indivíduos infectados ${ }^{4}$.

Os vetores triatomídeos habitam, no habitat humano, locais úmidos e escuros, muito comuns em casas desprovidas de recursos em alvenaria (as chamadas de "casas de pau a pique"). Tais residências são mais prevalentes em classes socioeconômicas menos favorecidas ou da zona rural, justificando o endemismo da Doença de Chagas nessas regiões ${ }^{4,6}$. Além disso, é possível a transmissão vertical via transplacentária, durante o parto ou, em alguns casos, durante amamentação ${ }^{6}$. Também pode ocorrer via oral, pela ingestão de alimentos contaminados pelos triatomíneos ou suas fezes, por via transfusional, por transplante de órgãos ou acidentes laboratoriais ${ }^{6}$.

Acredita-se que grande parte dos indivíduos infectados por doença de Chagas no mundo não possuem acesso a diagnóstico e tratamento adequado, elevando a morbimortalidade e o custo social da doença ${ }^{3}$. Pois, maior parte da população infectada possuí maior vulnerabilidade, sendo negligenciadas em diversas situações como menor cobertura por ações preventivas, menor acesso aos serviços de saúde ou atendimento de má qualidade na atenção primária a saúde e entre outros fatores ${ }^{3}$.

O objetivo desse trabalho é traçar o perfil epidemiológico das mortes pela doença de Chagas no estado do Tocantins no período de 2008 a 2018. Por se tratar de uma antropozoonose de elevada prevalência e significativa morbimortalidade no país, são essenciais os estudos acerca do assunto, entre os quais o conhecimento de seu perfil epidemiológico. Assim, pode-se estruturar a criação de projetos e tomadas de decisão pelo governo como envio de recursos públicos para prevenção da doença e tratamento dos

\section{METODOLOGIA}

\section{Coleta de Dados}

Este trabalho consistiu em uma pesquisa longitudinal, qualitativa e quantitativa, baseada em dados obtidos a partir de instituições governamentais (DATASUS ${ }^{8}$ e IBGE $^{9 a, b}$ ) para cada município do Tocantins, com respectivas macrorregiões de saúde, entre os anos 2008 e 2018. Os dados coletados do DATASUS foram: número absoluto de óbitos, totais (de todas as causas somadas) e relacionados à Doença de Chagas (CID-10 B57); número de médicos; número de agentes comunitários de saúde (ACS); e quantidade de Unidades de Vigilância em Saúde (UVS). Os registros de óbitos foram analisados discriminadamente, conforme município de residência, para: cada ano; ambos os sexos; etnia preta, parda, branca ou ignorada; faixas etárias de menor que 30 anos, 30-39 anos, 4049 anos, 50-59 anos, 60-69 anos, 70-79 anos e 80 anos ou mais; níveis de escolaridade ausente, 1-3 anos, 4-7 anos, 8-11 anos, 12 anos ou mais, ou ignorado; local de registro do óbito como hospital, outra instituição de saúde, domicílio, via pública, outros ou ignorado. Os dados registrados mensalmente pelo DATASUS foram coletados adotando-se como referência os registros de março de cada ano. Já as informações referentes à população dos municípios foram obtidas conforme estimativas do IBGE. A informação acerca da população beneficiária de Planos de Saúde no Tocantins foi acessada via ANS ${ }^{10}$, tomandose como parâmetro o registro de informações do mês de março de cada ano.

\section{Análise de Dados}

A Proporção de Agentes Comunitários de Saúde (PACS) foi calculada conforme sugestão do Ministério da Saúde ${ }^{11}$, no qual se recomenda a relação de um ACS para, no máximo, 750 habitantes. A Proporção de Médicos Generalistas (PMG) foi calculada pelo número total de médicos (independente da especialidade) para cada 1.000 habitantes, para cada município.

Realizaram-se, com os softwares Microsoft Office Excel ${ }^{\circledR}$ e IBM $^{\circledR}$ SPSS $^{\circledR}$ Estatistics 20.0, a análise descritiva e, para as variáveis não-paramétricas, os testes de comparação de dois grupos Wilcoxon-Mann-Whitney e de comparação de múltiplos grupos de Kruskal-Wallis. O nível de significância adotado foi de 0,05 ( $\alpha=5 \%$ ).

\section{RESULTADOS}

Os óbitos por Doença de Chagas no estado do Tocantins analisados no DATASUS no período de 2008 a 2018 foi de 578 . Dentre as cidades mais acometidas, estão Palmas e Araguaína. Observou-se uma tendência à diminuição no percentual de óbitos ao longo dos anos, mas a comparação da distribuição entre 2008 e 2018 não foi significativa ( $W=-1,275 ; p=0,202$ ). Nas figuras 1 e 2 podemos observar os dados dos óbitos registrados em comparação o número de UVS e o número relativo ACS/750hab, respectivamente. A correlação entre 
médicos/1000hab e a incidência de óbitos por Chagas, embora Figura 2. Média da porcentagem dos óbitos por ano devido a significativa estatisticamente, apresentou-se fraca $(\rho=0,146$; Doença de Chagas no Estado do Tocantins entre (2008-2018) $p<0,001)$.

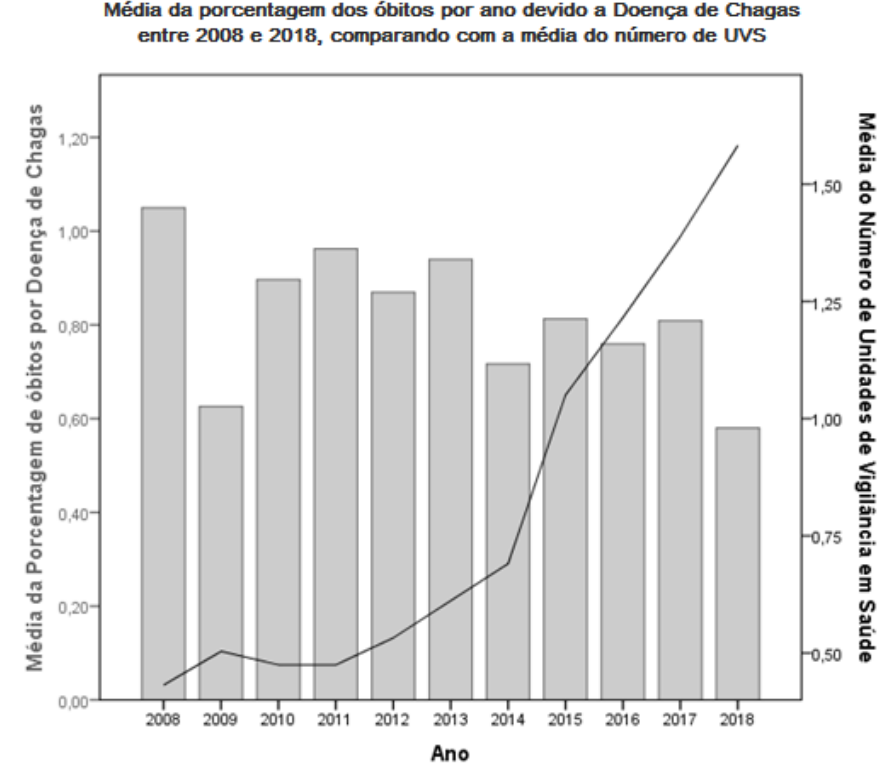

Figura 1. Média da porcentagem dos óbitos por ano devido a Doença de Chagas no Estado do Tocantins entre (2008-2018) (eixo da esquerda, barras cinzas), comparando com a média do número de UVS (eixo da direita, linha preta).

Observando a variável sexo, nota-se que houve um predomínio do sexo masculino com $60 \%$ em comparação com $40 \%$ do sexo feminino; $(U=112,500 ; p<0,001)$. No que diz respeito a cor de pele (Figura 3), a cor parda predomina com $57,96 \%$; cor branca 26,82\%; cor preta com $11,25 \%$ e ignorada com 3,98\%. Com relação a faixa etária (Figura 4), a idade entre 70 e 79 anos foi a mais acometida pela Doença de Chagas com 169 óbitos, seguidos das faixas entre 60 e 69 anos (151), 80 anos ou mais (141), 50 a 59 anos (77), 40 a 49 anos (29), 30 a 39 anos (11) e menos de 30 anos (0).

Média da porcentagem dos óbitos por ano devido a Doença de Chagas entre 2008 e 2018, comparando com a média do número de ACS por municipio

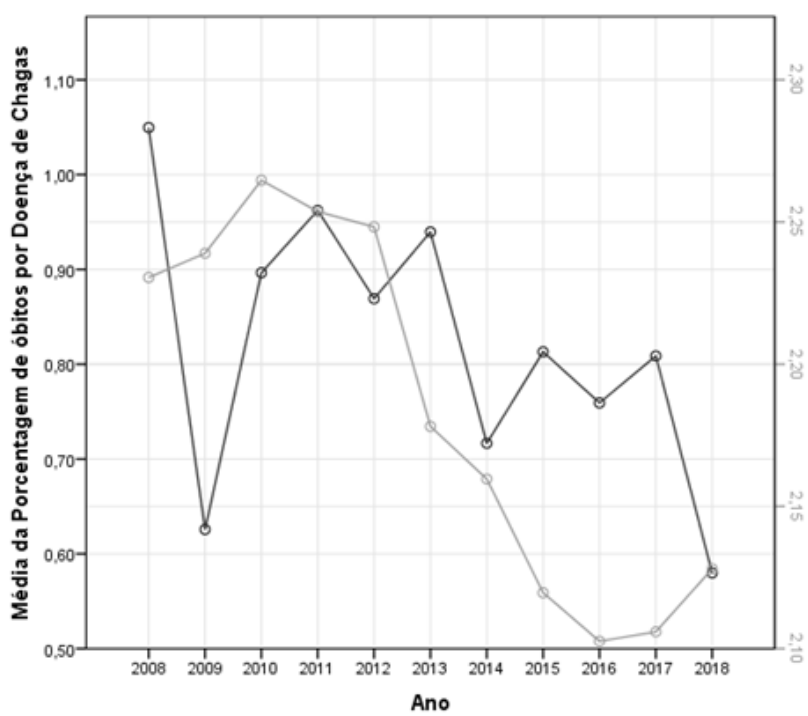
(eixo da esquerda, preto), comparando com a média do número de ACS por município (eixo da direita, cinza).

A partir da categoria 4 a 7 anos é notável como a incidência de óbitos diminui progressivamente na medida que o tempo de escolaridade aumenta $(\rho=0,020)$ (Figura 5). Levando em consideração o local de ocorrência, a maioria aconteceu no hospital $(67,82 \%)$, seguido do domicílio $(24,05 \%)$, outros $(3,46 \%)$, outro estabelecimento de saúde $(2,25 \%)$, via pública $(2,08 \%)$ e ignorado $(0,35 \%)(K=51,834 ; G L=5 ; p<0,001)$. Por fim, na Figura 6, observa-se a distribuição percentual de óbitos conforme a Macrorregião de Saúde.

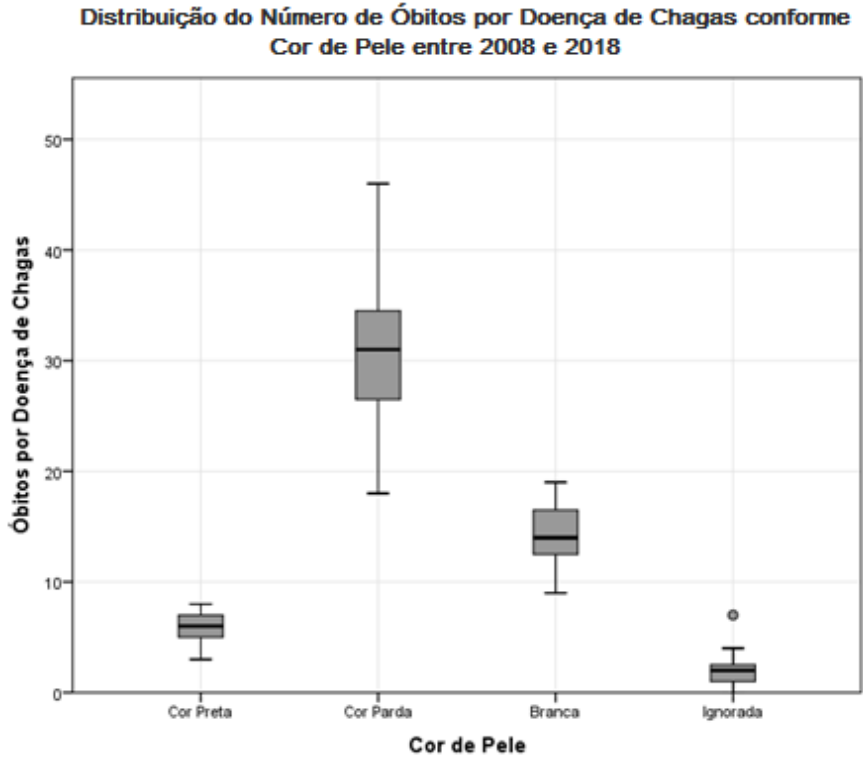

Figura 3. Distribuição dos óbitos por Doença de Chagas no (2008-2018) conforme a cor de pele; ( $K=38,880 ; \quad G L=3$; $\mathrm{p}<0,001)$.

Distribuição do Número de Óbitos por Doença de Chagas conforme Faixa Etária entre 2008 e 2018

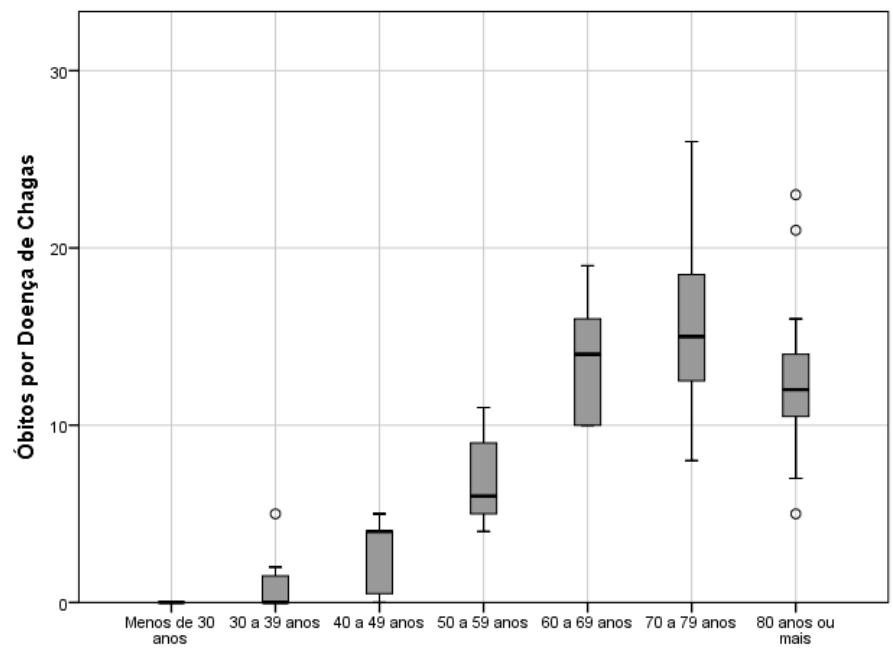

Faixa Etária

Figura 4. Distribuição dos óbitos por Doença de Chagas no Tocantins (2008-2018) de acordo com a faixa etária $(K=6,488$; $\mathrm{GL}=6 ; \mathrm{p}<0,001)$. 
Distribuição do Número de Óbitos por Doença de Chagas conforme o Tempo de Escolaridade entre 2008 e 2018

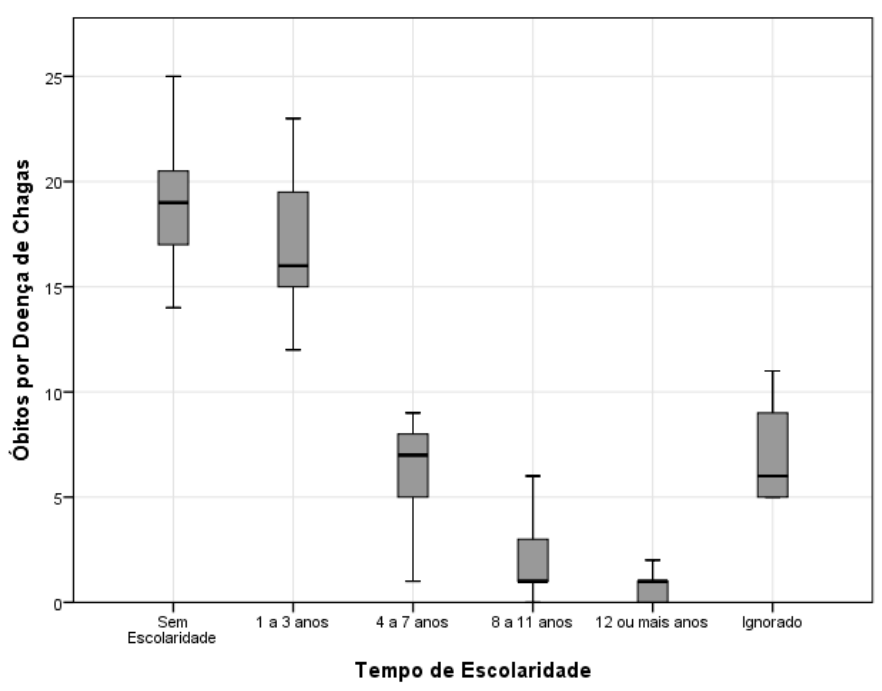

Figura 5. Distribuição do número de óbitos por Doença de Chagas no Tocantins (2008-2018) conforme tempo de escolaridade $(K=56,769 ; G L=5 ; p<0,001)$.

Distribuição do percentual de óbitos por Doença de Chagas conforme a Macrorregião de Saúde

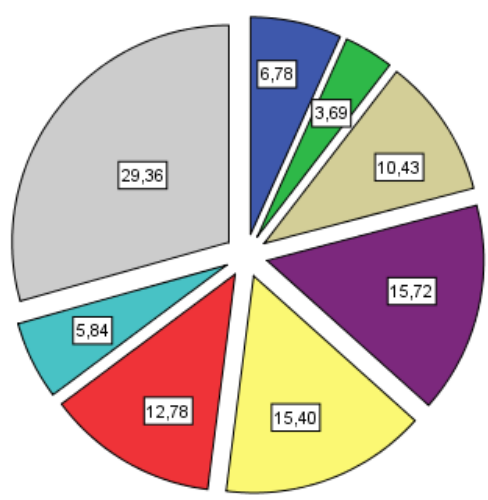

Macrorregião de Saúde $\square$ Amor Perfeito Bico do Papagaio Cantåo

Capim Dourado

Cerrado Tocantins-Araguai Illha do Bananal $\square$ Sudeste

Figura 6. Setores conforme o percentual de óbitos por Doença de Chagas no Tocantins (2008-2018) por Macrorregião de Saúde.

\section{DISCUSSÃO}

Ao analisar os casos de óbitos por Doença de Chagas compreendidos entre os anos de 2008 e 2018 nota-se uma estabilidade. Uma vez que o número de mortes se mantém estável, denota-se uma falha no acompanhamento desses doentes no serviço de saúde.

Além disso, outro fator importante é a maior ocorrência de óbitos em homens, abarcando quase $60 \%$ dos óbitos notificados. Tal fato está em consonância com os resultados encontrados por outros estudos ${ }^{12,13,14,15,16}$, que também demonstraram maior incidência no sexo masculino. Isso se dá por uma condição sócio-cultural em que o homem se vê como invulnerável e não procura o sistema de saúde, pois considera o autocuidado como uma prática feminina ${ }^{17}$. De forma geral, o homem é mais vulnerável às doenças, especialmente às crônicas, mantendo uma maior taxa de morbimortalidade, pois não buscam o sistema de saúde. Diante desta realidade o Ministério da Saúde, em 2008, instaurou a Política Nacional de Atenção Integral à Saúde do Homem, na tentativa de reduzir tais taxas ${ }^{18}$. Contudo ao analisar os dados levantados de mortalidade pela Doença de Chagas, nota-se que esta política ainda está longe de atingir seu objetivo.

A Doença de Chagas tem maior prevalência e maior gravidade em pacientes idosos, igualmente evidenciado na literatura ${ }^{12,13,14,15,16}$, uma vez que, por se tratar de uma doença crônica, na qual os efeitos deletérios vão surgindo ao longo dos anos, é compreensível que afete mais a população de idade mais avançada ${ }^{19}$. A doença apresenta duas fases, a primeira é uma fase aguda que acomete majoritariamente a população adulta jovem, apresentando sintomas mais leves ou moderados, contudo, posteriormente, ela entra em latência e pode desenvolver problemas cardíacos e digestivos ${ }^{20}$. Tendo isso evidente, é importante o fortalecimento de políticas de saúde que visem o tratamento e acompanhamento de pacientes jovens e idosos portadores de chagas, mas, principalmente, naqueles que estão na fase de latência para diminuir a progressão da doença e ter menos repercussões negativas quando chegarem na velhice, na tentativa de fornecer uma melhor qualidade de vida e diminuir a progressão da doença.

O número de Unidades de Vigilância de Saúde (UVS) parece estar relacionado aos casos de mortalidade por chagas, sendo que é possível notar uma relação inversa entre o número de UVS e o número de óbitos relativo a Chagas (Figura 1). Essa relação pode ser atribuída ao protocolo de tratamento, no qual os pacientes devem ser acompanhados pela equipe de atenção primária do seu território, apoiando os pacientes no tratamento e acompanhando a evolução da doença ${ }^{21}$.

Em contrapartida, um dado interessante que foi evidenciado é o fato de que a diminuição dos agentes comunitários de saúde (ACS) para cada 750 habitantes coincidiu com a diminuição de óbitos ocorridos na região. Sendo que os ACSs são responsáveis por ações de vigilância e controle, além da instrução da população no que tange à Doença de Chagas e de serem essenciais na notificação de insetos vetores. Contudo, percebese pelos dados que entre 2017 e 2018 houve um aumento no número de ACS que coincidiu com a queda de óbitos ${ }^{22}$. Alguns estudos ${ }^{23,24}$ demonstraram a deficiência de parcela dos ACSs no que se refere ao conhecimento sobre a Doença de Chagas e também com relação aos serviços de Atenção Primária para com essa doença. Talvez essa seja a razão de o aumento de ACS em todo o período apresentado não ser diretamente proporcional à queda de óbitos.

Outro dado digno de nota é que a escolaridade se mostrou diretamente associada ao acometimento pela doença. Isso pode se dar pelo fato do indivíduo com baixa escolaridade procurar empregos na área rural ou mesmo com condições inferiores, além de ser um fator social para a periferização das pessoas, fazendo com que a população possa entrar em contato maior com o barbeiro (inseto vetor). Ademais, pessoas com maior escolaridade tem maior propensão a procurar serviços de saúde do que pessoas com baixa escolaridade ${ }^{24}$. Diante disso, nota-se que o grau de instrução representa um fator protetivo, sendo que quanto maior a escolaridade, menores são as chances de ter um óbito por chagas.

Além disso, é possível perceber que a cidade com maior número de óbitos é a capital Palmas, pertencente à macrorregião Capim Dourado, local de maior ocorrência da doença. As demais cidades com alto número de óbitos não 
correspondem às respectivas regiões com maiores ocorrências. A partir disso nota-se que os óbitos estão distribuídos amplamente pelo estado do Tocantins. Segundo Souza ${ }^{15}$, a zona urbana concentra a maior parte da mortalidade com 50,61\%. Sendo assim, é importante a elaboração de ações de prevenção e acompanhamento da Doença de Chagas nas principais regiões, que são Capim Dourado, Sudeste e Ilha do Bananal, além de ações nas cidades específicas de Palmas, Araguaína, Porto Nacional e Gurupi com o intuito de reduzir a ocorrência da doença nesses locais e, consequentemente, diminuir o número de óbitos. É importante, ainda, ressaltar que tais medidas são urgentes, visto que somente as regiões Capim Dourado e Sudeste compreendem quase $50 \%$ das ocorrências de todo o estado, representando uma fragilidade no sistema de saúde dessas localidades no que tange ao tratamento da Doença de Chagas.

Ademais, é importante, ainda, salientar que, segundo o boletim epidemiológico divulgado pelo Ministério da Saúde em abril de $2020^{19}$, a taxa de óbitos no Tocantins entre os anos de 2010 e 2017 esteve maior que a taxa do Brasil em todos os anos. Tal referência não trazia informações sobre a taxa de 2018. Ainda é válido destacar que o Estado do Tocantins manteve uma taxa maior que a maioria dos estados neste mesmo período e apresentou a maior taxa da região Norte.

Por fim, foi evidenciado que grande parte dos óbitos ocorreram em âmbito hospitalar. Isso provavelmente se manifesta devido ao fato de que os óbitos por Chagas normalmente não são súbitos e, mesmo em casos graves/urgentes, o paciente é encaminhado aos hospitais ${ }^{26}$. A evolução crônica da doença de chagas pode levar ao desenvolvimento de insuficiência cardíaca congestiva ou mesmo cardite letal ${ }^{4}$. Além disso, os efeitos no sistema gastrointestinal também podem surgir com a cronicidade da doença. Assim, quando o paciente busca atendimento hospitalar, devido a progressão da doença, ele já se encontra em um estado muito debilitado e acaba indo à óbito em decorrência das complicações.

\section{CONCLUSÃO}

Os óbitos por doença de Chagas no Tocantins mantiveram-se estável durante o tempo entre 2008 e 2018, mesmo com o aumento de medidas públicas como criação de UVS. Sua maior incidência compreendeu as macrorregiões de saúde Sudeste, Capim Dourado e Cerrado Tocantins-Araguaia, principalmente em seus respectivos hospitais. O perfil do paciente caracterizase por predominantemente por indivíduos do sexo masculino, da cor parda, entre 70 e 79 anos e baixa escolaridade. Nota-se a necessidade de reavaliação e elaboração de programas de ações de saúde mais efetivos que permitam a redução na mortalidade por Doença de Chagas no Estado do Tocantins.

\section{REFERÊNCIAS BIBLIOGRÁFICAS}

1. World Health Organization. Research priorities for Chagas disease, human African trypanosomiasis and leishmaniasis. WHO: technical report of the TDR Disease Reference Group on Chagas Disease, Human African Trypanosomiasis and Leishmaniasis. Geneva: World
Health Organization; 2012. (WHO Technical Report Series, 975).

2. World Health Organization. Sustaining the drive to overcome the global impact of neglected tropical diseases: second WHO report in neglected tropical diseases. Geneva: World Health Organization; 2013.

3. Dias JCP, Ramos Júnior NA, Gontijo ED, Luquetti A, Shikanai-Yasuda MA, Coura JR, et al. II Consenso Brasileiro em doença de Chagas, 2015. Brasília: Epidemiologia e Serviços de Saúde; 2016.

4. Kumar V, Abbas AK, Fausto N, Mitchell RN. Robbins. Bases patológicas das doenças. 8. Ed. Rio de Janeiro: Elsevier, 2010.

5. Ministério da Saúde (Brasil). Doença de Chagas: o que é, causas, sintomas, tratamento e prevenção. Disponível em: https://antigo.saude.gov.br/saude-de-a-z/doencade-

chagas\#: :text=A\%20doen\%C3\%A7a\%20de\%20Chagas\%2 0(ou,\%2C\%20card\%C3\%ADaca\%2C\%20digestiva\%20ou\%2 Ocardiodigestiva.

6. Ministério da Saúde (Brasil). Guia de Vigilância em Saúde, 2 ed. Brasília, DF: O Ministério; 2017.

7. Ministério da Saúde (Brasil). Portaria no. 264, de 17 de fevereiro de 2020. Altera a Portaria de Consolidação no 4/GM/MS, de 28 de setembro de 2017, para incluir a doença de Chagas crônica, na Lista Nacional de Notificação Compulsória de doenças, agravos e eventos de saúde pública nos serviços de saúde públicos e privados em todo o território nacional. Diário Oficial da União 17 fev 2020; Seção 1.

8. Ministério da Saúde (Brasil). DATASUS. Disponível em: http://www2.datasus.gov.br/DATASUS/index.php?area $=0$ 204\&id=6906.

9. IBGE, Instituto Brasileiro de Geografia e Estatística. Estimativas da População.

10. IBGE, Instituto Brasileiro de Geografia e Estatística. Censo de 2010. Disponível em: https://www.ibge.gov.br/estatisticas/sociais/populacao/ 9662-censo-demografico-2010.html?=\&t=resultados.

11. Ministério da Saúde (Brasil). ANS, Agência Nacional de Saúde Suplementar. Dados e Indicadores do Setor.

12. Ministério da Saúde (Brasil). PNAB, Política Nacional de Atenção Básica. 1a Edição, 110p. Brasília, DF. 2012.

13. Litvoc J, Wanderley DMV, Camargo LMA. Mortalidade por doença de Chagas no Estado de São Paulo (Brasil): subsídios para o planejamento da assistência ao chagásico. Rev. Saúde Pública. 1992 Apr; 26 (2): 59-65. Disponível em: https://doi.org/10.1590/S003489101992000200001.

14. Bozelli CE, Araújo SM, Guilherme ALF, Gomes ML. Perfil clínico-epidemiológico de pacientes com doença de Chagas no Hospital Universitário de Maringá, Paraná, Brasil. Cad. Saúde Pública [Internet]. 2006 May; 22 (5): 1027-1034. Disponível em: https://doi.org/10.1590/S0102-311X2006000500015.

15. Santo $A H$. Tendência da mortalidade relacionada à doença de Chagas, Estado de São Paulo, Brasil, 1985 a 
2006: estudo usando causas múltiplas de morte. Rev

Panam Salud Publica. 2009; 26 (4): 299-309.

16. Souza CNP, et al. Fatores contribuintes à ocorrência de mortalidade por doença de chagas. Rev. Bras. Biom., São Paulo, v. 32, n. 4, p.544-552, 2014.

17. Moraes CA. Mortalidade por doença de chagas no estado de Goiás, Brasil, no período de 2006 a 2011, Cicílio Alves de Moraes. 2017. xiv. $52 \mathrm{f}$.

18. Gomes R, Nascimento EF, Araújo FC. Por que os homens buscam menos os serviços de saúde do que as mulheres? As explicações de homens com baixa escolaridade e homens com ensino superior. Cad. Saúde Pública. 2007 Mar; 23 (3); 565-574. Disponível em: https://doi.org/10.1590/\$0102-311X2007000300015.

19. Ministério da Saúde (Brasil). Secretaria de Políticas de Saúde. Política Nacional de Atenção Integral à Saúde do Homem. Brasília (DF); 2008.

20. Ministério da Saúde (Brasil). Secretaria de Vigilância em Saúde. Doença de Chagas: 14 de abril - Dia Mundial. Bol Epidemiol. 2020 abr; 51 (n. esp.): 1-43.

21. Ministério da Saúde (Brasil). Secretaria de Políticas de Saúde. Coordenação-Geral de Desenvolvimento da Epidemiologia em Serviços. Guia de Vigilância em Saúde: Volume Único. Brasília (DF); 2008.

22. Organização Pan-Americana da Saúde. Doença de Chagas. Guia para Vigilância, prevenção, controle e manejo clínico da doença de chagas aguda por alimentos.

23. Ministério da Saúde (Brasil). Secretaria de Vigilância em Saúde. Guia de Vigilância Epidemiológica | Caderno 10; Doença de Chagas.

24. Flores MS. Análise do Conhecimento sobre Doença de Chagas dos Agentes Comunitários de Saúde dos Municípios de Abaetetuba e Tailândia, Estado do Pará, Brasil. Rio de Janeiro, 2016.

25. Rodrigues FCS. Agentes comunitários de saúde: percepção sobre os serviços de saúde relacionados à doença de Chagas. Cad. saúde colet. 2020 Mar; 28 (1): 130-139. Epub Apr 09, 2020. Disponível em: https://doi.org/10.1590/1414-462×202000280458.

26. Barata RB. A posição social e seus reflexos sobre a saúde. In: Como e por que as desigualdades sociais fazem mal à saúde. Rio de Janeiro: Editora FIOCRUZ, 2009. Temas em Saúde collection, pp.23-39. ISBN 978-85-7541-391-3. Available from SciELO Books.

27. França SB, Abreu DMX. Morbidade hospitalar por doença de chagas no Brasil. Revista da Sociedade Brasileira de Medicina Tropical, 29 (2): 109-115, mar-abr, 1996 